\title{
Leveraging Digital Technology to Transform Accounting Function: Case Study of a SME
}

\author{
Gary Pan (Corresponding author) \\ School of Accountancy, Singapore Management University, Singapore \\ E-mail: garypan@smu.edu.sg
}

Benjamin Lee

School of Accountancy, Singapore Management University, Singapore

E-mail: benjaminlee@smu.edu.sg

Received: May 19, 2020

Accepted: June 22, 2020

Published: June 30, 2020

doi:10.5296/ijafr.v10i2.17052

URL: https://doi.org/10.5296/ijafr.v10i2.17052

\begin{abstract}
Digital transformation is taking hold of the processes and systems in accounting at a rapid pace and in a drastic way. With its emphasis on data and analysis, accounting entity is well suited to reap the benefits digital technologies have to offer. With the rapid infusion of digital technologies in accounting entity, the accounting literature has been calling for more empirical studies to examine the way accounting function leverages digital technology to enhance its analytical capabilities so as to obtain deeper business insights. This paper conducts an empirical study to examine the process of a SME's accounting function leveraging data analytics to enhance its financial analysis capability and revenue growth prediction in developing its future business strategy. In this study, we have used Lewin's (1951) change theory as our analytical perspective. We have drawn on Food Co's data analytics adoption experience by interviewing relevant internal stakeholders and reviewing secondary data extensively. Our analysis identified three stages of "unfreeze-change-refreeze" digital transformation process and several intervention actions that were instrumental in enacting the implementation of data analytics platform.
\end{abstract}

Keywords: Digital technology, Digital transformation, Accounting, Small and medium-sized enterprises 


\section{Introduction}

In order to meet the needs of dynamic business environment and evolving consumer preferences, many companies have embraced digital technologies to transform their organizational structures, processes and service/product offerings (Berman, 2012). These digital technologies include real-time data analytics, robotic process automation, artificial intelligence (AI) and others. What these companies are undergoing is the phenomenon of digital transformation. Digital transformation is the process of using digital technologies to create new - or modify existing - business models and process, application and tools, culture, and customer experiences to meet changing business and market requirements. For digital transformation to succeed, companies need to conceptualize how new digital technology may affect current business processes and models (Kane et al., 2015), invest and deploy appropriate organizational resources, and most importantly, develop capabilities to turn digital technologies into digital innovation to enhance business processes and strengthen decision-making (Fichman and Melville, 2014).

Digital transformation of organizations is occurring not only in the core operational areas along the value-added chain, but also in the central functions such as purchasing, human resources and accounting. In particular, it is taking hold of the processes and systems in accounting at a rapid pace and in a drastic way. This should not come as a surprise as accounting is generally known to be heavily reliant on data and analysis. As such, the accounting function is well suited to reap the benefits digital technologies have to offer. With digitalisation, complex accounting transactions using traditional methods will now be processed easily and quickly. Some examples of digitalisation in accounting involve real-time reporting, process automation, data visualisation, big data analytics and cloud computing. With rapid technological development, the accounting literature has been calling for more empirical studies to examine the way accounting function leverages digital technology to enhance its analytical capabilities so as to obtain deeper business insights (Kruskopf et al., 2019).

Small and medium-sized enterprises (SMEs) are more likely to benefit from digital innovation than their larger and more established counterparts (Chan et al., 2019), which are often reluctant to adopt new digital innovation for fear of losing the foundation of their existing competitive advantages (Christensen, 1997). However, the adoption by SMEs often meets with challenges as SMEs are disadvantaged by resource limitation when it comes to embracing digital innovation (Meister, 2017). Therefore, it is valuable to learn more about ways SMEs could adopt emerging technologies to enhance its business processes and decision-making mechanisms.

To address the knowledge gaps in both Accounting and SME literature, this paper conducts an empirical study to examine the process of a SME's accounting function leveraging data analytics to enhance its financial analysis capability and revenue growth prediction in developing its future business strategy. In our case selection, we have selected a Singapore based company called Food Co (pseudonym) who has successfully leveraged data analytics to enhance its capability in accounting function. 


\section{MInstitute ${ }^{\text {Macrothink }}$}

International Journal of Accounting and Financial Reporting

ISSN 2162-3082

2020, Vol. 10, No. 2

In terms of theoretical lens, this paper drew upon Lewin's (1951) theory of change to examine the adoption process of data analytics. In particular, we focused on the change process of how Food Co experimented with a new digital idea, explored its feasibility and came to a consensus to adopt and turn data analytics into financial analytical capability. We view Lewin's theory as an appropriate theoretical lens for this study because in many 'technology entrapment situations', there are competing forces of change which may encourage adoption or resistance of new technology (Teger, 1980). The 'unfreezing-changing-refreezing' lens helps to analyze these competing forces of change, which tend to create a conflict over the decision to adopt or resist a technology entrapment situation.

The remainder of the paper is organized as follows: We first review the digital transformation literature. We then outline how Lewin's theory of change can act as a theoretical lens to make sense of a digital transformation process. This is followed by a description of our research approach and our case study, Food Co's accounting function journey in embracing data analytics. Following the case study, we apply Lewin's framework in our analysis and present some useful findings. The paper ends with conclusion, and implications for research and practice.

\section{Literature Review}

\subsection{Digitalisation in Accounting and SMEs}

There are three phases of digital transformation: digitization, digitalization, and digital transformation. Digitization describes encoding of analog information into a digital format (Loebbecke and Picot, 2015) and it mainly digitalizes internal and external documentation processes without changes in value creation. Digitalization describes how digital technologies can be used to alter existing business processes ( $\mathrm{Li}$ et al., 2016). Through digitalization, firms apply digital technologies to optimize existing business processes, hence allowing a more efficient coordination between processes that may enhance customer experiences (Pagani and Pardo, 2017). Digital transformation describes a company-wide change that leads to the development of new business models, which may improve its competitive advantage and create value (Pagani and Pardo, 2017). Digital transformation rearranges the processes, routines and capabilities, and changes the business logic of a firm (Li et al., 2018).

While digital transformation has been observed as broad-scale institutional change in numerous industries (e.g., retail, transportation, accommodation, finance, or legal services), the accountancy sector is just on the cusp of this revolution. Accounting is on its way to make a great change of the role it plays in the organization and the functions it currently performs. The proponents of digital revolution view this transformation as a step-ahead and embrace the challenges of the future. Existing accounting literature highlights that the accounting function can benefit significantly from digital transformation. For example, Issa et al. (2016) suggest that accounting activities and processes are primed for automation owing to their laborious tasks and wide range of decision structures. Loh and Ashton (2019) also highlight that the accounting function can drive productivity through automation of processes, which 
may bring about increased efficiency, improved decision-making, and an enhanced control environment.

Prior studies have identified a few challenges of implementing digital transformation. For instance, Loh and Ashton (2019) highlight several factors that contribute to the failure of process automation projects. In particular, they highlight the lack of a clear RACI (Responsible, Accountable, Consulted, and Informed) framework, getting the execution wrong and lack of ongoing technology management as key reasons for such failures. In is therefore important to gain insights into how an accounting function may successfully adopt digital technologies to enhance its processing and analytical capability.

According to Chan et al. (2019), SMEs are typically less formal, lean, and flatter in terms of their organizational structure in comparison to large corporations. Given their inherent size limitation, they tend to operate at a smaller scale using focused and specialized resources, capabilities, and processes catering to their niche/specific markets, customers, and industry domain (Meister, 2017). Most of them do not have the capabilities to develop digital-ready processes (Neirotti and Raguseo, 2017). Levy and Powell (2000) suggest that senior management who are open to innovations and changes to their internal and external processes are more likely to adopt IT innovation. It is also believed that SMEs who chose to adopt IT innovation and align their internal processes with these innovations tend to perform better than those that do not (Cragg et al. 2002).

Overall, our review highlights a knowledge gap in the literature in that little is known about the evolution of an accounting function of a SME, leveraging digital technology to enhance its processes and capabilities. It is therefore the objective of our paper to advance knowledge by plugging this gap in the accounting literature.

\subsection{Adapting Lewin's Theory of Change}

To achieve our objective in this research, we adapted Lewin's (1951) theory of change as a theoretical lens to help us understand the change process during digitalisation. Lewin's (1951) theory of change can be used as a lens to conceptualise the inertias and enablers of how internal stakeholders in an organization experiment new ideas, explore their feasibility or alternative ideas and eventually come to a consensus to accept new ideas. Generally, the theory evolves around a basic change model of unfreezing, changing, and refreezing. The model perceives human change as a 'profound psychological dynamic process that involves painful unlearning without loss of ego identity, and difficult relearning as one cognitively attempts to restructure one's thoughts, perceptions, feelings and attitudes' (Schein, 1996, p. 27). In addition, the model also suggests change as a multistage process and all stages must be negotiated before a stable change can take place (Schein, 1988).

Figure 1 shows Lewin's stages - a transformation process framework we adapt for guiding our study. The framework suggests that unfreezing can only take place when there is motivation to change, and such motivation could be either self-induced or influenced by peers. Unfreezing tends to generate a certain extent of psychological struggle within individuals, which can often be inherently painful. The change process involves the development of new 
ideas based on new information and cognitive redefinition. Refreezing can only begin when new ideas are adopted. Finally, before relearning stabilizes, there must be successful alignment and integration with individuals' values and beliefs. Applying Lewin's theory of change to examine the transformation process of digitalisation will help us to achieve the objective of this study.

$\begin{array}{lll}\text { Stage 1: Unfreezing } & \text { Stage 2: Changing } & \text { Stage 3: Refreezing } \\ \text { Creation of Motivation } & \text { Developing New Attitudes } & \text { Stabilizing the Changes } \\ \text { to Change } & \begin{array}{l}\text { and Behaviours on the Basis } \\ \text { of New Information and }\end{array} & \\ & \text { Cognitive Redefinition } & \end{array}$

Step 1:
Disconfirmation of
Expectations

Step 2: Induction of Learning Anxiety

Step 3: Provision of Psychological Safety

$\begin{array}{ll}\text { Step 1: Identification with a } & \text { Step 1: Align and } \\ \text { Role Model and Seeing } & \text { Integrate the New } \\ \text { Things from that other } & \text { Attitudes and Behaviours }\end{array}$

Person's Point of View

Step 2: Scanning the

Environment for

Step 2: Assess Whether

Others will Accept and

Confirm the New

Information Specifically

Attitudes and Behaviour

Relevant to One's Particular

Problem

Figure 1. A transformation process framework (Adapted from Lewin, 1951)

\section{Research Methodology}

Our strategy was to undertake qualitative case research of a data analytics project at Food Co. We approached our fieldwork at Food Co, with a premise that data analytics adoption undergoes a transformation process and identifiable using an existing theoretical lens. Accordingly, our study draws upon Lewin's (1951)'s transformation process framework to examine Food Co's data analytics adoption process.

The research access was negotiated with Food Co in January 2018. From January to June 2018, we conducted our data collection. Primarily, semi-structured interviews were conducted with relevant project stakeholders. These semi-structured interviews were taped-recorded with interviewees' permission and transcribed immediately after the meetings. Altogether, 11 interviews were conducted, each lasted an average of one to one-and-a-half hours involving 7 interviewees. The interviewees include Chief Financial Officer (CFO), Human Resource Manager, Sales Manager, Accounting Executives and Sales Executives. The interview questions were prepared prior to the interview sessions. These questions were aimed at identifying the critical factors that enacted the transformation process during data analytics project implementation. Interviewees represent various "voices" (Qu \& Dumay, 2011). Secondary data such as reports and memos were also gathered to supplement the 
information collected through interviews. To ensure the reliability of our data, the interview data were triangulated with other secondary data for validation.

Data analysis was carried out by recursively iterating between the empirical data, the theoretical lens, and the relevant literature. The iterative process continued until the state of theoretical saturation was reached, that is, when it was possible to comprehensively explain the findings of the case study and no additional data needed to be collected or added to improve the findings. We began our analysis by reading all transcripts and documents and highlighting the descriptions that were related to the change process during digitalisation. We used analytic induction technique (Taylor and Bogdan, 1998) to uncover new constructs and relationships that could enrich our understanding of the phenomenon and assist our theory building process. Analytical induction involved the following steps (Goetz and LeCompte, 1981): (1) A phenomenon is defined in a tentative manner, (2) scanning data to identify categories, (3) developing categories, (4) determining the relationships that exist among categories, and (5) continually refining categories until all are accounted for.

We then developed a list of common themes. Coding categories reflect our interpretations of the critical factors in the change process. An initial pilot run was conducted for coder training and pilot testing of reliability. During the pilot run, coding instrument and procedures were also refined. To establish the reliability of the coding, each coder was asked to quote a particular segment of the relevant texts. Coding was conducted independently and without consultation and guidance. We examined the portions of the codings where both coders agreed and measured the inter-coder reliability using Cohen's Kappa coefficient. Our coefficient score of 0.79 suggests substantial agreement between the two coders and the result also demonstrates that the categories were clearly defined, and could be located in the text with little ambiguity. As the reliability coefficient was high, each coder was subsequently asked to code separate portions of the texts. Relevant interview comments and secondary reports were sorted according to the various categories and a list of themes within each category was developed. The list contained the location of each comment on the transcript, the transcript number, the interview date, any links to other comments and reports. In order to reduce researcher bias, a senior colleague was asked to take part in early analysis of some of the data. The colleague was uninvolved in the fieldwork and was therefore unfamiliar with Food Co's case. The role of this colleague was to bring a different and possibly more objective eye to the evidence and detect any bias in data analysis.

\subsection{Case Study - Food Co}

Food Co's main line of business was the manufacturing and processing of rice cracker snacks. Food Co's competitive positioning lay in offering quality rice cracker products at affordable prices. Besides selling at the retail market, Food Co also provided private label packaging for local house brands; and contract manufacturing for the service industries, such as hotels and airlines.

Food Co covered an extensive range of over 10 product flavours, including original, sesame, peanuts, cheese, chilli and others. Original flavour was Food Co's bestseller. Food Co's rice crackers were selling in both local and overseas markets. In 2015, the company began to 


\section{Mll Macrothink}

International Journal of Accounting and Financial Reporting

ISSN 2162-3082

2020, Vol. 10, No. 2

export its rice crackers overseas; and as at 2019, Food Co could be found in 8 countries in Asia.

The opportunities for businesses in the food industry to tap on data analytics were numerous. For example, supermarkets may analyse consumer buying preferences from past sales trends and use the insights derived from the analyses to stock its inventory level such that it matches demand in a timely manner, thereby increasing customer satisfaction. The other areas where data analytics may support the making of critical business decisions include new product development, investigation of supplier or production issues, formulation of product pricing and promotion strategies.

While there are many benefits in adopting data analytics, several SME business owners feel intimidated using data analytic capabilities due to lack of knowledge. They simply do not know how they could leverage their existing data to make better decisions. Another obstacle that has impeded data analytics adoption is the low priority of allocating budgets in SMEs for long-term planning and data analysis.

\subsection{Food Co’s Data Analytics Journey}

Food Co's aspiration was continual growth. With the aim, it had to capture larger market shares and make debuts in new markets. According to the CFO who joined Food Co in early 2017 ,

When I first joined, the biggest challenge was that I did not have a tool to help me understand our data.

Food Co had accumulated a voluminous amount of data over the years. On product information alone, the company had to keep track of more than 10 varieties of retail products in different packaging designs and weight. For example, the rice crackers came in re-sealable and single-use packs, small cans, bottles and gift boxes during festive periods. The packs were available in retail stores as small party packs of $40 \mathrm{~g}$, handy packs of $125 \mathrm{~g}, 130 \mathrm{~g}$ and $150 \mathrm{~g}$, standard packs of $300 \mathrm{~g}$ and $400 \mathrm{~g}$, bigger family packs of one kilogram and so on.

In addition to the various retail packaging formats were the customisations for private labelling, again in different packaging, weight and quantity. The Sales Manager explained,

Every customer had her own requirements. We emphasised on customer service and became so accommodating to all our customers that it created a problem for us. We had too many stock keeping units (SKUs) to manage. We also had to hold so much raw materials and packaging materials. Different customers wanted different ways of packaging, and it led to wastage. It became a big headache.

Managing the SKU for each product became a phenomenal task. The CFO soon realised that insightful discoveries from data were needed to address the SKU problem. Remarked CFO,

If you don't have the correct tool to help you understand where you are going and what you will need to get to it, you will never succeed. 


\section{Macrothink}

International Journal of Accounting and Financial Reporting

ISSN 2162-3082

The CFO decided to engage a business consultant to design and implement data analytic platform. Even though Food Co's accounting executives admitted that data analytics would offer insights to their business data, nevertheless, they were reluctant to embrace data analytics, as none of them knew much about data analytics and how to use the data analytical tools. As one of the accounting executives mentioned:

We had no idea how to use any of the data analytics tools. Even with training,

I wasn't confident if I could master those tools within a short time.

Knowing users' anxiety, the CFO assured everyone in the accounting department that adequate training would be provided to all accounting executives. As part of the design process, the consultant would also seek everyone's input in the design of the data analytics platform. In addition, the CFO assured the team that he too, did not know enough of data analytics and would be joining everyone in the training sessions. His assurance managed to calm the accounting executives and relieve their anxiety. Besides, Food Co also offered study leave for the accounting staff undergoing the data analytics training workshops. According to the Human Resource Manager, staff would be more willing to sign up for the workshop if study leave was provided as an incentive. By June 2017, work on the data analytics project had begun.

The big deterrence to further global expansion was the absence of important supporting information, such as operating costs, return-on-investment, production quantity, and so on. This information was a must to assess the potential risk of investing in different foreign markets, and its absence ultimately determined the overall expansion strategy. Thus a key value proposition of data analytics was that it could help Food Co visualise what the future held, and hence in justifying decisions made. According to the CFO,

Data analytics could give us some light into the unchartered way. The beauty about data was that it showed us what we had been doing all this while, and going forward, we won't do it the same way. The data could also tell us what will happen next.

The aim of the data analytics platform was to support Food Co in their decision making process towards strategy planning, drive brand awareness for new products as well as maintain customer loyalty for existing ones. Once the accounting staff and Sales Manager understood data analytics could predict potential sales markets, they became more convinced about adopting the data analytics tools.

Using Food Co's historical sales data, the analysis revealed seasonal buying patterns by local consumers. It also showed the products which were most responsive during those periods. For instance, a spike in consumer demand was reported during the festive months of December to February every year, which coincided with Christmas, New Year and Chinese New Year celebrations. During these festive periods, original and sesame flavours were the most popular rice crackers in the local market. 
Acting on these patterns, Food Co could manage the supply chain process more efficiently during the festive period by adjusting the procurement of raw materials, and managing production, marketing, distribution and warehousing suitably to cater to the increased demand.

While little attention had been paid to demand forecasting in the past, it was believed that the introduction of predictive analytics could assume an important role in influencing the way Food Co planned its production schedule. The Sales Manager had wanted to instil an understanding of customer buying behaviour in his sales staff by observing trends in the sales data. In order to facilitate the active tracking of actual sales, he devised a 12-week monitoring plan. He shared,

Since I look at my sales record every week, by the fifth week I would have seen it for the fifth time, and I know what will happen thereafter. I can tell that we will get this certain amount of sales for the coming week.

With improved accuracy in sales forecast, production would be more responsive to actual demands. Eventually, Food Co would be able to revise their production schedule from a monthly to weekly cycle, as a shorter cycle could potentially increase production efficiency.

Food Co's global expansion strategy had set its sights on Middle East. Its strategy was to expand existing markets and to penetrate new markets. The Sales Manager elaborated,

We were trying to target markets we were big in, and the markets we were not yet in. We had good presence in several parts in Asia. We really wanted to go into the Middle East market, but we know it was very competitive.

Breaking into new markets would need special attention and a careful evaluation of the financial impact of introducing new products. Added the CFO,

For example, India is an exporter of rice, so rice are very cheap there. We could not bring rice crackers into India. We didn't really have a competitive edge. Our predictive results confirmed our knowledge.

Using predictive analytics, it was hoped that Food Co would be able to calculate the likelihood of success when introducing a new product in a new market. The predictive model suggested countries that had market potential for rice cracker consumption, and based on the preferred manufacturing quantity, it would also calculate the start-up costs, the sales volume that would enable Food Co to breakeven, the return-on-investment and the expected profit following five years of operations.

Launching new products into existing markets could prove equally challenging, given the plausible risk of cannibalisation on the profits of current products in the same category. The Sales Manager illustrated,

In Thailand, for example, we were already selling original rice crackers. If we came up with a new tom yam flavour, and the customer liked it, he or she would not buy original flavour anymore. 
Another capability of the predictive model was to manage the above challenge by quantifying the potential reduction in sales and revenue of well-established products upon the market introduction of a new product variant under the same family brand.

Beaming with satisfaction as he used his data analytics tool, the CFO and the Sales Manager thought aloud,

The data analytics tool helped us to predict how long it takes to achieve our targets.

Armed with data insights, the CFO was more confident about conquering new foreign markets, as demand could now be predicted with some degree of accuracy. He would share his new-found insights with the sales and marketing departments, and with the other group functions including HR and operations. With the newly acquired data analytical capabilities, The Human Resource Manager foresaw it would no longer be business-as-usual for Food Co. He mused,

This data analytics project also allowed me to better plan my manpower. I guess it's all about getting the business to the next level.

\section{Case Analysis and Findings}

We know that digitalisation is a complex process (MaryAnne, 2018) and actors play key roles in facilitating the digitalisation strategy. By applying the steps provided by the framework shown in Figure 1, we analyzed in the following section how actors in Food Co embraced digital processing and analysis of data in its accounting function.

\section{Stage 1: Unfreezing - Creation of Motivation to Change}

Schein (1996) suggests that unfreezing basically involves three processes: (a) disconfirmation of expectations, (b) induction of learning anxiety if the disconfirming data are accepted as valid and relevant, and (c) provision of psychological safety that converts anxiety into motivation to change. Lewin (1951) views that status quo would change easily if restraining forces such as personal defences and group norms were unfrozen.

In the case of Food Co, the gap between the CFO's expectation when he first joined the company and the way the accounting function was operating, was huge. Even though Food Co had accumulated voluminous amount of data over the years, the CFO lamented he did not have a tool to assist him to understanding those data. In a way, the CFO disagree with the lack of data analysis or the previous approach of handling data. For example, the CFO mentioned, "Data analytics could give some light into the unchartered way. The beauty about data was that it showed us what we had been doing all this while, and going forward, we won't do it the same way. The data could also tell us what will happen next." Basically, the $\mathrm{CFO}$ and the Sales Manager felt they could not obtain any insight from the voluminous data to manage its SKUs and diverse requirements from various clients.

The CFO's decision to engage a business consultant to design and implement data analytic platform sent a clear signal that the previous approach had failed to live up to his expectation. 


\section{Mll Macrothink}

International Journal of Accounting and Financial Reporting ISSN 2162-3082

The CFO's intervention was considered a vital disruption to group norms of previous way of handling data within Food Co.

The data also suggest that when the accounting executives were informed of the plan to adopt data analytics within the accounting function, there was significant level of anxiety among accounting users. User resistance that owes to fear of failure to master new knowledge, is viewed a negative emotional response which has been found to interfere with individuals' allocation of attention in the processing of new information. For instance, one of the accountants admitted, "We had no idea how to use any of the data analytics tools. Even with training, I wasn't confident if I could master those tools within a short time."

Subsequently, the anxiety was relieved when the CFO provided the necessary assurance and 'safety net' over the lack of data analytics skill among Food Co's accounting executives. His intervention helped raise the importance of the data analytics platform and also encouraged more participation among the accounting executives. Furthermore, the admission of the CFO in not knowing a lot about data analytics also reduced the accounting executives' fear of being penalised by Food Co. It is clear the creation of psychological safety has certainly helped to encourage accounting executives to participate actively in the digitalisation effort. Furthermore, it is also important that accounting executives were adequately motivated not only to attend training, but also involved in giving input during the design phase of the data analytics platform. A useful lesson learnt in the case is that one way to instil motivation and enthusiasm in project stakeholders is to provide adequate assurance (Heng et al., 2003).

\section{Stage 2: Changing - Developing New Attitudes and Behaviours on the Basis of New Information and Cognitive Redefinition}

Previous research (Grimley et al., 1994) suggests that when people are exposed to change interventions, they are at one of four stages: precontemplation, contemplation, action and maintenance. The organizational development literature suggests that the triggers of change come from at least five sources: the environment, performance, characteristics of top managers, structure, and strategy (Huber and Glick 1993). In many situations, people develop new attitudes by identifying with a role model (Schein, 1988). Here, the role of the change agent is that of prime mover who creates change.

In the case of Food Co, the challenge for the Consultant was to work with the CFO to obtain buy-in from the accounting staff and the Sales Manager to adopt the data analytics platform. For example, the CFO convinced the accounting executives and Sales Manager that data analytics could boost Food Co's desire for growth. This is because data could offer insights to what Food Co had been doing all this while, and predictive analytics could offer directions of future trends. The change went through a series of cognitive restructurings and semantic redefinitions. Once the accounting staff and Sales Manager understood that data analytics could predict future potential sales trends and markets, they became totally convinced about data analytics. The predictive model could support Food Co in its decision making process towards strategy planning, drive brand awareness for new products as well as maintain customer loyalty for existing ones. For example, both the CFO and the Sales Manager were 
pleased with the model and commented, "The data analytics tool helped us to predict how long it takes to achieve our targets."

The CFO and the Consultant pointed out new possibilities and set a new perspective. This triggered the change as the whole accounting function and sales team began to explore the potential of data analytics platform. These internal stakeholders started to cooperate and work towards a common adoption goal. The end result was the adoption of data analytics, which significantly enhanced its data analysis and predictive capabilities. It is important to highlight the important roles played by the CFO and the Consultant in instilling buy-in and commitment of the internal stakeholders.

\section{Stage 3: Refreezing - Stabilizing the Changes}

Refreezing assumes that the change process becomes a sequence of events in which "a person (a) determines or defines what currently exists, (b) determines or defines its replacement, (c) engages in action to remove what is currently there, and (d) implants its replacement" (Ford and Ford, 1994, p. 773). Beer et al. (1990) highlight that refreezing is difficult as integrating new attitudes and behaviours seem challenging simply because belief is a powerful sentiment and sometimes this 'faith' can be so hard to kill (Royer, 2003).

In the case of Food Co, the CFO was aware that full support and complete buy-in of the accounting executives and the Sales Manager held the key to the success of the digitalisation process. He encouraged a cohesive culture by identifying the users and consulted their opinions on the design of the data analytics platform. The Consultant also promoted teamwork by gathering the whole function and Sales Manager to brainstorm on the user requirements of the platform. By adopting a consultative approach, the $\mathrm{CFO}$ helped to legitimize the new course of action by making it a joint decision among the users and management - a refreezing process. Overall, these influence tactics deployed by the CFO and Human Resource Manager entice the users to overcome the commitment to the existing course of action and persuade them to accept new course of action - the data analytics platform. According to the Human Resource Manager, "This data analytics project also allowed me to better plan my manpower. I guess it's all about getting the business to the next level."

\section{Implications and Conclusion}

The purpose of our paper has been to conduct an empirical study to examine the process of a SME's accounting function leveraging data analytics to enhance financial analysis and revenue growth prediction in developing its future business strategy. In this study, we have used Lewin's (1951) change theory as our analytical perspective. We have drawn on Food Co's data analytics adoption experience by interviewing relevant internal stakeholders and reviewing secondary data extensively. Our analysis identified three stages of "unfreeze-change-refreeze" digital transformation process and several intervention actions that were instrumental in enacting the implementation of data analytics platform.

From research point of view, our paper makes a significant contribution to the accounting literature by examining the digitalisation process of a SME's accounting function, a context 


\section{Mll Macrothink}

International Journal of Accounting and Financial Reporting

ISSN 2162-3082

2020, Vol. 10, No. 2

that is little known. Through our case study, we have uncovered how users could be influenced to buy in to digitalisation through specific actions and strategies undertaken.

From managerial point of view, our study may offer a stark reminder for SMEs to overcome their resistance to digitalisation. A common challenge in digitalisation for SMEs is that many are still operating as traditional, non-digital businesses. They are greatly inhibited by three main barriers to adoption. Owing to a lack of digital and technical knowledge, these SME owners feel intimidated by the idea of adopting digital technologies (technical barrier). They are not able to envisage how digital technologies could be leveraged to help them make better business decisions and improve business performance (behavioural barrier). These two barriers further inhibit the design and development of domain-specific tools that value-add to the business (design barrier). As a consequence, these SMEs set a lower priority for investing in digitalization when it comes to budget allocation.

For SMEs to embark on digitalization, our findings have emphasized the need for SMEs to expand and improve their internal capabilities in achieving advantageous performance outcome. During the initial stage of digitalisation, management should collect user feedbacks to assess users' needs and constraints, and a detailed analysis of the resources and capabilities of the organization. Strategies must be developed to overcome the constraints, especially during the initial stage. The transformation of leadership into collective leadership during the adoption phase would require senior management to be actively involved in changing the mindset and actions of relevant internal stakeholders. Leadership can be enacted and transformed through championing, i.e. visibly advocating for the implementation and exemplarily providing role model for relevant stakeholders.

Future research is needed to re-examine the intervention actions uncovered in this study in other digitalisation contexts. As in any social process, there may be other issues that play their part in shaping the outcome. For instance, the case study in this research is that of a SME's accounting function. It would be interesting to study other types of SME entities, in which the organizational structures may differ in nature.

\section{References}

Beer, M., \& Nohria, N. (2000). Breaking the Code of Change. HBS Press. Boston, MA.

Berman, S. J. (2012). Digital Transformation: Opportunities to Create New Business Models. Strategy \& Leadership, 40(2), 16-24.

Business Network. Industrial Marketing Management, 67, 185-192.

Chan, C., Teoh, S. Y., Yeow, A., \& Pan, G. (2019). Agility in Responding to Disruptive Digital Innovation: Case Study of a SME. Information Systems Journal, 29(2), 436-455.

Christensen, C. M. (1997). The Innovator's Dilemma: When New Technologies Cause Great Firms to Fail. Harvard Business School Press, Boston, MA.

Cragg, P., King, M., \& Hussin, H. (2002). IT Alignment and Firm Performance in Small Manufacturing Firms. Journal of Strategic Information Systems, 2, 109-132. 


\section{$\triangle$ Macrothink}

International Journal of Accounting and Financial Reporting

ISSN 2162-3082

Fichman, R. G., \& Melville, N. P. (2014). How Posture-Profile Misalignment in IT Innovation Diminishes Returns: Conceptual Development and Empirical Demonstration. Journal of Management Information Systems, 31, 203-239.

Ford, J., \& Ford, L. (1994). Logics of Identity, Contradiction, and Attraction in Change. Academy Management Review, 19, 756-785.

Goetz, J., \& LeCompte, M. (1981). Ethnographic Research and the Problem of Data Reduction. Anthropology and Education Quarterly, 12, 51-70.

Grimley, D., Prochaska, J., Velicer, W., Blais, L., \& DiClemente, C. (1994). The Transtheoretical Model of Change. In Brinthaupt, M. (Ed.), Changing the Self: Philosophies, Techniques, and Experiences (pp. 201-207). New York: State University. NY Press.

Heng, C., Tan, B., \& Wei, K. (2003). De-escalation of Commitment in Software Projects: Who Matters? What Matters?. Information \& Management, 41(1), 99-110.

Huber, G., \& Glick, W. (1993). Organizational Change and Redesign. New York: Oxford University Press.

Issa, H., Sun, T., \& Vasarhelyi, M. (2016). Research Ideas for Artificial Intelligence in Auditing: The Formalization of Audit and Workforce Supplementation. Journal of Emerging Technologies in Accounting, 13(2), 1-20.

Kane, G. C., Palmer, D., Phillips, A. N., \& Kiron, D. (2015). Is Your Business Ready for a Digital Future?. Sloan Management Review, 56, 37-44.

Kruskopf, S., Lobbas, C., Meinander, H., Söderling, K., Martikainen M., \& Lehner, O. M. (2019). Digital Accounting: Opportunities, Threats and the Human Factor. ACRN Oxford Journal of Finance and Risk Perspectives, 8, 1-15.

Levy, M., \& Powell, P. (2000). Information Systems Strategy for Small and Medium Sized Enterprises: An Organizational Perspective. Journal of Strategic Information Systems, 9, 63-84.

Lewin, K. (1951). Field Theory in Social Science. Harper and Row, New York.

Li, F., Nucciarelli, A., Roden, S., \& Graham, G. (2016). How Smart Cities Transform Operations Models: A New Research Agenda for Operations Management in the Digital Economy. Production Planning \& Control, 27(6), 514-528.

Li, L., Su, F., Zhang, W., \& Mao, J. Y. (2018). Digital Transformation by SME Entrepreneurs: a Capability Perspective. Information Systems Journal, 28(6), 1129-1157.

Loebbecke, C., \& Picot, A. (2015). Reflections on Societal and Business Model Transformation arising from Digitization and Big Data Analytics: A Research Agenda. Journal of Strategic Information Systems, 24, 149-157. 


\section{$\triangle$ Macrothink}

International Journal of Accounting and Financial Reporting ISSN 2162-3082

Loh, M., \& Ashton, D. (2019). Using Intelligent Automation to Transform the Finance Function. In Goh, C., Pan, G., Seow, P. S., Lee, B., \& Yong, M. (Eds.), Charting the Future of Accountancy with AI (2019). CPA Australia: Singapore.

MaryAnne, G. (2018) Digitalization, Digitization, and Innovation. Research Technology Management, 61(4), 56-59.

Meister, D. B. (2017). Entrepreneurial Firms and Information Systems Capabilities. Twenty-Third Americas Conference on Information Systems.

Neirotti, P., \& Raguseo, E. (2017). Flexible Work Practices and the Firm's Need for External Orientation: An Empirical Study of SMEs. Journal of Enterprise Information Management, 30, 922-943.

Pagani, M., \& Pardo, C. (2017). The Impact of Digital Technology on Relationships in a

Qu, S., \& Dumay, J. (2011). The qualitative research interview. Qualitative Research in Accounting \& Management, 8(3), 238-264.

Royer, I. (2003, February). Why Bad Projects Are So Hard to Kill?. Harvard Business Review, 49-56.

Schein, E. (1988). Organizational Psychology. Prentice Hall, Eaglewood Cliffs, NJ, United States.

Schein, E. (1996). Kurt Lewin's Change Theory in the Field and in the Classroom: Notes Toward a Model of Managed Learning. System Practice, 9, 27-47.

Taylor, S., \& Bogdan, R. (1998). Introduction to Qualitative Research Methods (3rd ed.). Wiley.

Teger, A. (1980). Too Much Invested to Quit. Pergamon, New York.

\section{Copyright Disclaimer}

Copyright for this article is retained by the author(s), with first publication rights granted to the journal.

This is an open-access article distributed under the terms and conditions of the Creative Commons Attribution license (http://creativecommons.org/licenses/by/4.0/) 